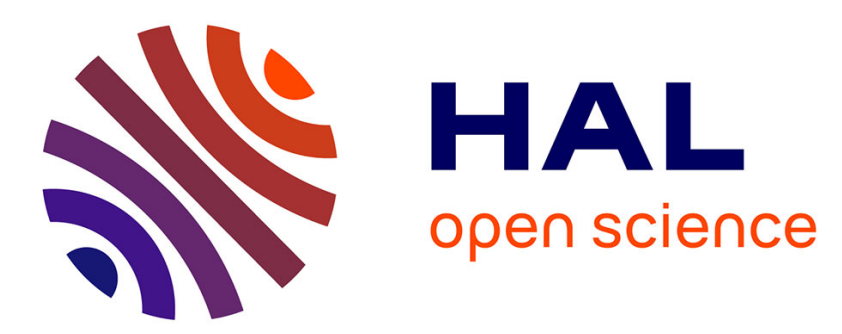

\title{
Transition from deep to shallow water layer: formation of vortex dipoles
}

\author{
Damien Sous, Natalie Bonneton, Joël Sommeria
}

\section{To cite this version:}

Damien Sous, Natalie Bonneton, Joël Sommeria. Transition from deep to shallow water layer: formation of vortex dipoles. European Journal of Mechanics - B/Fluids, 2005, 24, pp.19-32. 10.1016/j.euromechflu.2004.06.002 . hal-00260775

\section{HAL Id: hal-00260775 \\ https://hal.science/hal-00260775}

Submitted on 27 Mar 2020

HAL is a multi-disciplinary open access archive for the deposit and dissemination of scientific research documents, whether they are published or not. The documents may come from teaching and research institutions in France or abroad, or from public or private research centers.
L'archive ouverte pluridisciplinaire HAL, est destinée au dépôt et à la diffusion de documents scientifiques de niveau recherche, publiés ou non, émanant des établissements d'enseignement et de recherche français ou étrangers, des laboratoires publics ou privés. 


\title{
Transition from deep to shallow water layer: formation of vortex dipoles
}

\author{
Damien Sous $^{\mathrm{a}, *}$, Natalie Bonneton ${ }^{\mathrm{b}}$, Joel Sommeria $^{\mathrm{c}}$ \\ a TREFLE, ENSCPB, 16, av. Pey Berland, BP 108, 33607 Pessac cedex, France \\ b L3AB, observatoire de Bordeaux, 2, rue de I'Observatoire, BP 89, 33270 Floirac, France \\ ${ }^{\mathrm{c}}$ LEGI/CORIOLIS, 21, avenue des Martyrs, 38000, Grenoble, France
}

In this paper we study the transition from a deep to a shallow water layer and the formation of quasi-two-dimensional vortex dynamics. Vortices are experimentally generated by a circular horizontal turbulent pulsed jet. The dimensional analysis gives two relevant dimensionless parameters: the jet Reynolds number $R e$ and a number $C$ which characterizes the vertical confinement. They are respectively defined by $R e=\sqrt{Q} / v$ and $C=\left(\sqrt{Q} / H^{2}\right) t_{\text {inj }}$ ( $H$ is the water depth, $v$ is the kinematical viscosity, $Q$ the injected momentum flux and $t_{\text {inj }}$ the injection duration). Experimental results show a strong influence of $C$ on the flow: when $C<1$, the evolution is a typical three-dimensional turbulence decay, while when $C>2$ we observe the formation of large vortex dipoles. However these dipoles are not strictly two-dimensional because of the presence of a vertical circulation in the front of the dipole. Results are independent of the jet Reynolds number in the range $1000<R e<1800$. In addition, a comparison between experimental shallow water dipoles and theoretical model is exposed.

Keywords: Vortex dipole; Quasi-two-dimensional turbulence; Shallow water; Experiment

\section{Introduction}

An experimental characterization of the transition from a deep to a shallow water layer is presented in this paper. By shallow water layer we mean a layer bounded by a no-slip bottom and a free surface in which the vortex structures size is large compared to water depth [1,2]. This definition has to be distinguished from the shallow-water model where water depths are much smaller than the surface wave horizontal length scales. Here, we consider the flow velocities sufficiently small to avoid surface excitation. Our experiments aim at understanding the influence of the vertical confinement in a test case: the vortex dynamics produced by an impulsive jet in an horizontal water layer. We especially focus on the transition between the deep water and the shallow water turbulence behavior.

Many water flows in coastal zones, lakes or rivers are constrained by the vertical confinement of their environment. As exposed by Jirka [3], observations have revealed the presence of large scale vortices in geophysical shallow flows. Due to the proximity of these shallow water flows with human activities and their contributions in geophysical processes (sediments transport and mixing processes), it is of great importance to improve our knowledge of the role of vertical confinement. Among shallow water flows, rip currents represent a particular application of the present work. These strong off-shore directed currents are induced indirectly by breaking waves [4]. In most cases, their formation is episodic and their vertical expansion is limited

\footnotetext{
* Corresponding author. Tel.: +33-5-40-00-33-07; fax: +33-5-40-00-66-68.

E-mail address: sous@enscpb.fr (D. Sous).
} 
by the free surface and the bottom. Hence, they can be assimilated to impulsive jets in shallow water. Their development is not fully understood because the three-dimensional flow structure is inconsistent with the classical description used by coastal engineers, obtained on the basis of a depth-integrated flow model [5].

Surprisingly, few researchers have worked on the turbulence behavior in homogeneous shallow water. Most of these studies have been carried out experimentally on plane continuous jets [6] and plane wakes [7]. In their experiments on turbulent continuous plane jets Dracos et al. [6] have shown the quasi-two-dimensionality of the flow in the far field (beyond ten times the depth), with the periodic formation of large counter-rotating structures and an energy spectra with a -3 wave-number dependence. Turbulent plane wakes in shallow water have been studied by Chen and Jirka [7]. In these experimental studies, the flow is controlled the Reynolds number $\operatorname{Re}_{H}=U H / v$ and the parameter $S=c_{f} D / H$ ( $c_{f}$ is the bottom friction coefficient defined by the shear velocity $u_{*}=\sqrt{c_{f} / 2} U_{a}$, where $U_{a}$ is the depth-averaged ambient velocity, $D$ the body width and $\mathrm{H}$ the depth). Hence, $S$ describes the confinement applied on the turbulent wake. When $\operatorname{Re}_{H}>1500$, Chen and Jirka have shown the flow stability is uniquely dependent on $S$.

Experiments on mixing layers in shallow water have also shown a particular behavior due to the shallowness [1,8]. The energy spectra identify the simultaneous presence of several turbulence scales in the flow: a Q2D turbulence with large horizontal vortices and small-scale motions on the solid bottom. For the shallower experiments, the large vortices are destroyed and the flow is uniquely controlled by the turbulent boundary layer. The development of a grid turbulence in a shallow water layer has been recently studied by Uijttewaal et al. [9]. Large Q2D vortices appear in the grid wake and are progressively destroyed by small-scale turbulence production on the solid bottom.

A condition for the flows quasi-two-dimensionality in a thin fluid layer has been proposed by Dolzhanskii et al. [10]. Q2D flows are controlled by two dimensionless parameters, namely the traditional Reynolds number $\operatorname{Re}_{v}=U L / v$ and the Reynolds number in terms of external friction $R e_{\lambda}=U / \lambda L$ (where $U$ and $L$ are respectively the characteristic velocity and length scales of the flow and $\lambda$ is the 'external friction' term defined by $\lambda=2 v / H^{2}$ for a thin fluid layer of thickness $H$ ). In the case $R e_{\lambda} \ll R e_{\nu}$, Dolzhanskii has shown that there is no dependence in $R e_{\nu}$. Moreover, the condition of quasi-two-dimensionality itself is equivalent to the above inequality, $R e_{\lambda} \ll R e_{\nu}$.

The influence of similar dimensionless parameters has been evaluated in a specific case by Satijn et al. [11]. By numerical simulation, they study the evolution of an axisymmetric circular vortex in a domain bounded by a no-slip bottom and a free surface. The dimensionless parameters are defined from the typical vorticity. They are the external Reynolds number $R e_{\lambda}=\omega / \lambda$ (where $\omega$ is the typical vertical vorticity and $\lambda=\pi^{2} v / 4 H^{2}$ is the external friction parameter with $H$ the height of the layer and $v$ the kinematical viscosity) and the structure Reynolds number $\operatorname{Re}=L^{2} \omega / v$ ( $L$ represents the typical horizontal length scale in the flow). Since the typical vorticity can be evaluated as the ratio of typical velocity divided by the typical length scale of the vortex, these dimensionless numbers are similar to those presented by Dolzhanskii [10]. By varying $R e$ and $R e_{\lambda}$, Satijn et al. obtained a regime diagram from which we can note that the quasi-two-dimensionality of the monopolar vortex is increased for small $\operatorname{Re}$ and $\operatorname{Re}_{\lambda}$. It reveals as expected that the vertical confinement increases the two-dimensionality of the flow.

Emergence of Q2D vortices have been observed in various situations. Many laboratory experiments performed on vortices in rotating fluids, stratified fluids and magneto-hydrodynamic flows have revealed this Q2D vortex dynamics.

Experiments in rotating fluids have shown the emergence of two-dimensional vortices [12]. In particular, Velasco Fuentes and van Heijst [13] have experimentally studied the propagation of dipolar vortices on a topographic $\beta$-plane. Measurements have shown the nonlinearity of the vorticity-stream function relationship. Moreover, the fluid rotation modifies the bottom boundary layer (appearance of the Ekman layer) and the dipole trajectory which is deflected towards the anticyclonic direction [14].

The effect of fluid stratification has been studied in numerous laboratory experiments. In particular, experimental investigations performed by Flòr and van Heijst [15] and Voropayev et al. [16] have detailed the formation of quasi-twodimensional (Q2D) laminar dipolar structures from an initially three-dimensional (3D) turbulent patch. The injection of small volume of fluid in stratified water at rest creates a localized three-dimensional turbulent flow region. The turbulent patch generated by the impulsion is quickly constrained by the density stratification. It collapses in large horizontal vortical structures, typically "pan-cake" dipoles. Most of these dipoles present a good agreement with the 2D theoretical model of Lamb and Chaplygin [17,18] (circular shape, positions of the vorticity extrema). However, by contrast with the model hypothesis, the relationship between the vorticity and the stream function is nonlinear in most cases. Voropayev et al. [16] studied the dipolar structure in terms of injected momentum. They gave a substantial attention to the entrainment of ambient fluid into the expanding dipole, for which they developed a theoretical model. The basic assumption of this model, identical to the Lamb and Chaplygin model, is that the motion inside the vortex dipole is similar to the steady invisible flow induced by two vortices, while the outside flow is the potential flow around a cylinder. A good agreement between the model and the experimental results has been obtained.

Q2D vortex dipoles have been also observed in the experiments carried out by Nguyen Duc and Sommeria [19]. The quasitwo-dimensionality of the flow has been obtained by applying an uniform magnetic field on a thin layer of mercury. The dipoles were found to have the same global characteristics as their counterparts observed in stratified fluids: circular shape, steady states characterized by a function $\omega=f(\psi)$ (where $\omega$ is the vorticity and $\psi$ is the stream function) such as its derivative $f^{\prime}$ is constant 
or has a singular maximum at the vortex core, and also possible interactions between symmetric dipoles by exchange of their vortices.

An extreme confinement can lead to the formation of two-dimensional vortices. This was nicely demonstrated by the soapfilm experiments of Couder and Basdevant [20]. They have observed the formation of dipolar structures in the wake of a thin cylinder that was towed through the film.

Thus, experiments in stratified [15,16] or rotating fluids [12,13] and soap films [20] have revealed the quasi-two-dimensional behavior of the turbulence, characterized by the emergence of large coherent vortices. The dipoles frequently observed in these flows have been widely studied and compared with theoretical 2D model.

Shallow flows can also develop a Q2D dynamics, characterized by emergence of large coherent vortices, [1,6,8,9,11]. However, any experimental characterization of the transition from deep to shallow flows is lacking. Our study aims at understanding the role of vertical confinement in a test case: the vortex dynamics produced by an impulsive jet. We focus our attention on the free evolution of an initially $3 \mathrm{D}$ turbulence generated by an impulsive turbulent jet in a homogeneous shallow water layer. Existing research on shallow jets has been performed on plane jets [6], so the initial geometry is two-dimensional. By contrast, in our experiments a fluid impulsion through a cylindrical nozzle initially generates a fully 3D homogeneous turbulent patch, so a transition from 3D to Q2D flow can be studied. In particular, we would like to answer the following questions: can we identify a specific "shallow water behavior"? Where is the transition between the shallow and deep water? Can an impulsive turbulent jet in a shallow water layer develop into Q2D dynamics? Are there any analogies between vortex dynamics in shallow water and in stratified or rotating fluids?

In order to answer these questions, the first step is to determine the dimensionless parameters which control the flow.

\section{Dimensional analysis}

We use a small injector nozzle, and study the jet at distance large in comparison with the nozzle diameter. Because of the turbulent jets self-similarity [21], the jet diameter $d$ is not a pertinent parameter, the expansion rate is constant and the jet development depends only on the distance to the "virtual origin" of the jet. This virtual origin is located inside the injection nozzle, at the convergence point of the jet boundaries. For a turbulent pulsed jet, the two pertinent parameters are the injected momentum flux $Q$ [16] and the injection duration $t_{\text {inj }}$. Thus, four independent parameters describe our experiments:

$-v$, the kinematic viscosity $\left[\mathrm{m}^{2} \mathrm{~s}^{-1}\right]$,

- $Q$, the injected momentum flux given by $Q=(U S)^{2} / S=U^{2} S\left[\mathrm{~m}^{4} \mathrm{~s}^{-2}\right]$,

- $H$, the water depth $[\mathrm{m}]$,

- $t_{\text {inj }}$, the injection duration $[\mathrm{s}]$,

where $U$ is the injection speed and $S$ the nozzle section.

From these parameters, two dimensionless numbers can be defined. First, the jet Reynolds number $R e$, based on the injected momentum flux, is given by:

$$
R e=\frac{\sqrt{Q}}{v}
$$

We have called the second dimensionless number $C$ the confinement number, defined by:

$$
C=\frac{\sqrt{Q}}{H^{2}} t_{\text {inj }}
$$

$C$ characterizes directly the vertical confinement applied on the pulsed jet: the greater $C$ is (which corresponds to large injected volume or small depth), the stronger the confinement applied is.

The time (observation time from the injection) $t$ is also a preponderant parameter. Every quantitative measurement is carried out for dimensionless times $t^{*}=t / t_{\text {inj }}$ between $t^{*}=6$ and $t^{*}=7.5$, which are small compared to the time necessary to the total viscous dissipation of motions. Such times $t^{*}$ are large enough to ensure that the jet is sufficiently developped to enable the flow structuration, and small enough to avoid the tank boundaries influence.

In order to understand the influence of $C$ and $R e$ on the vortex dynamics, qualitative and quantitative investigations have been carried out with the experimental setup described in Section 2. The parametrical study results are presented in Section 3 for the qualitative observations and in Section 4 for the quantitative measurements. Main features of dipoles in shallow water layer are described in Section 5. Finally, a discussion of the results and conclusions are proposed in Section 6. 


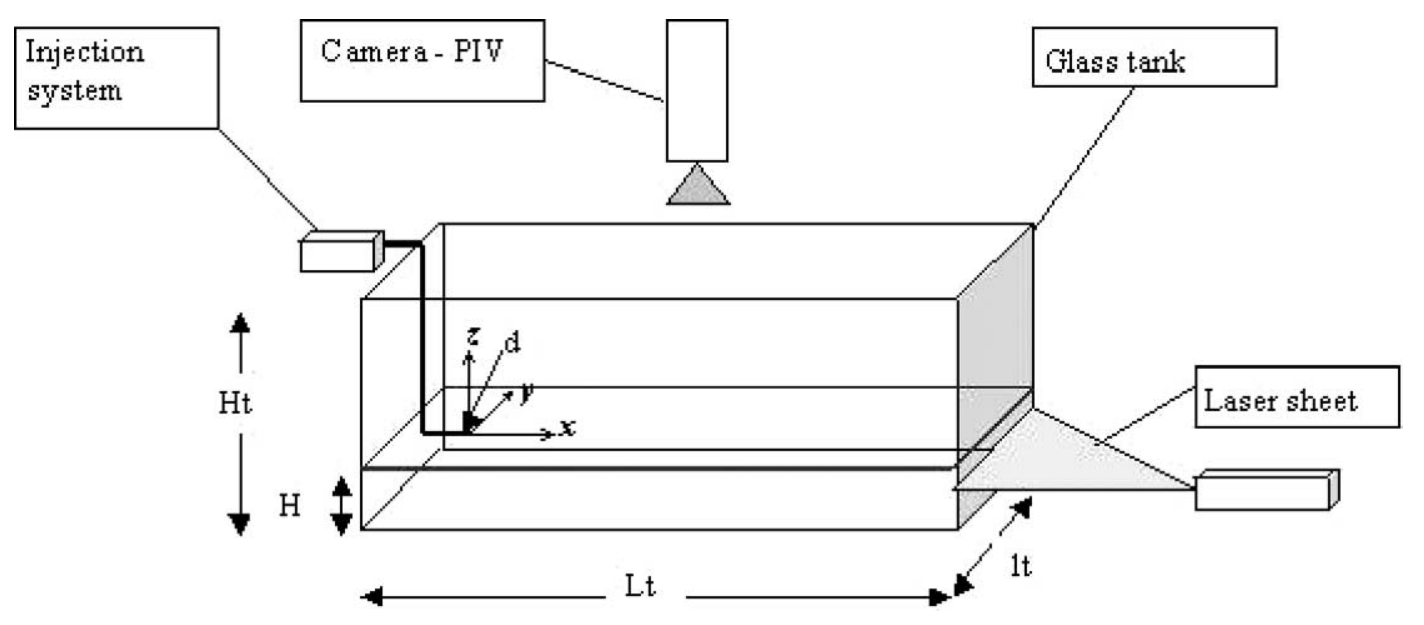

Fig. 1. Sketch of the experimental setup.

\section{Experimental setup}

Experiments have been performed in a glass tank $\left(H_{t}=1 \mathrm{~m}, L_{t}=0.6 \mathrm{~m}, l_{t}=0.5 \mathrm{~m}\right)$, see Fig. 1 . We inject horizontally a small amount of fluid through a cylindrical nozzle in water at rest. During our experiments, four parameters are modified: the depth, the nozzle diameter, the injection speed and the injection duration. The depth $H$ varies from 1 to $20 \mathrm{~cm}$, the nozzle diameter $d$ from 1 to $1.7 \mathrm{~mm}$, the injection speed from 0.5 to $2.5 \mathrm{~m} / \mathrm{s}$ and the injected duration from 1.8 to $14 \mathrm{~s}$. Depending on the injection speed and duration, the injected volume varies from 1 to $15 \mathrm{ml}$. The injected fluid is taken directly from the glass tank to have the same properties. The injection duration is controlled by an electric gate. The injection speed is controlled by varying the hydrostatic pressure and measured by Particle Image Velocimetry. It is held constant during the injection duration.

\subsection{Flow visualizations}

Two visualization techniques have been used in our experiments to obtain qualitative information on the flow.

Firstly, in order to follow the global flow evolution, fluoresceine is used to track the injected fluid, using a laser sheet illumination (argon laser). Secondly, the streamlines are visualized by particles streaks: nylon particles (size $50 \mu \mathrm{m}$, density $1100 \mathrm{~kg} \mathrm{~m}^{-3}$ ) are added to the whole fluid (injected fluid and fluid contained in the tank).

\subsection{Quantitative measurements}

Quantitative measurements have been performed by Particle Image Velocimetry (PIV). PIV is a nonintrusive velocity measurement technique which provides instantaneous velocity fields. It consists in analyzing the displacements of particles added to the flow.

The PIV system used for the present study is developed by TSI. The light sheet illumination is provided by a Spectra Physics argon laser (S-P 2550 2016-05), with a wave length of $600 \mathrm{~nm}$ (green light). It emits a continuous circular light beam, with a typical power of $4 \mathrm{~W}$. This circular beam is carried by an optical fiber and converted into a light sheet with a thickness of $1 \mathrm{~mm}$ at the optical fiber exit. A CCD camera (TSI 630044-A 4-30 $10 \mathrm{~Hz}$ RS-170) with a $640 \times 480$ resolution is used. The camera captures frames with a $33 \mathrm{~Hz}$ frequency. Due to the moderate velocities, we have to take at least a 10-frame sequence to obtain sufficient particles displacements. In the particular case of high velocity measurements in the near nozzle jet, a laserpulse has been used (T.S.I 620010 Beammodulator). It is composed of rotating mirrors synchronized with the camera to have access to higher measurement frequencies than the camera frame rate.

The step following the frame grabbing is the correlation process. A two-frame cross correlation algorithm has been used. It consists in a comparison between the particle distributions of two successive frames. The two frames are divided into a number of interrogation areas. In our measurements, the size of these interrogations areas has been chosen between $16 \times 16$ and $48 \times 48$ pixels with an overlap between 25 and $50 \%$. The software Insight from TSI is used to analyze the raw data and extract the velocity field.

The seeding of the flow is of great importance for the quality of PIV measurements. The particles need to accurately follow the local flow. It means that the particles should have a density very close to that of the fluid and a small size to avoid perturbations. The seeding density (particle concentration) is governed by the need to have roughly 10 particles per interrogation 
region. This density should be homogeneous in the whole observed region. In our measurements, we have used pliolite particles, with a size about $50 \mu \mathrm{m}$ and a density of $1100 \mathrm{~kg} \mathrm{~m}^{-3}$. Salt has been added to the water to match the particle density.

The global uncertainties on PIV velocity measurements are about $5 \%$.

\section{Qualitative observations}

The first step of our work was to identify, by qualitative observations, the influence of the dimensionless parameters on the flow evolution.

\subsection{Confinement number influence}

In order to depict the fundamental difference between dynamics evolutions in shallow and deep water, two representative cases are compared: $C=3$ for the shallow water case, and $C=0.18$ for the deep water case. In Fig. 2 , two sequences of pictures represent the compared time evolutions of the turbulent patch from the injection. The nozzle and the laser sheet are disposed horizontally at half depth. The left column shows the evolution in shallow water $(C=3)$ and the right column in deep water $(C=0.18)$. Both sequences have been taken from the same observation area $\left(60 \times 46 \mathrm{~cm}^{2}\right)$ and at the same instants $(t=0.2 \mathrm{~s}$, $5 \mathrm{~s}, 10 \mathrm{~s}, 20 \mathrm{~s}$ and $30 \mathrm{~s})$. Since the injection duration is the same in both cases $t_{\mathrm{inj}}=3 \mathrm{~s}$, the dimensionless time $t^{*}=t / t_{\mathrm{inj}}$ takes the following values: $0.07,1.7,3.33,6.67$ and 10 .

At the initial time (just after injection) $t^{*}=0.07$, the flow is similar for both cases: a compact and intense turbulent patch (jet Reynolds number $R e=1300$ ). From $t^{*}=1.7$, a strong difference between both dynamics appears.

- For $C=0.18$, the turbulence remains 3D. Typical size of the vortex structures increases slowly. The vortices are progressively stretched with a nearly isotropic motion. Their intensities decrease with time until they finally decay by viscous dissipation.

- For $C=3$, the behavior is different. The turbulent patch is not stretched, it remains compact. At $t^{*}=1.7$, the vortices size stays moderate, but the flow remains compact, there is no vortex stretching. At $t^{*}=3.33$, the smaller vortices disappear whereas a dipolar vortex structure merge at the front of the flow. After $t^{*}=3.33$, the small scale turbulence still decay with time, whereas the dipole size progressively increases. Finally, the dipoles typical lengthscale is at least ten times greater than the depth. The fluid layer can then be considered shallow, according to our definition based on the vortex structure size.

Fig. 3 shows streaks of particles added to the flow for the cases $C=3.3$ and $C=0.2$. The Reynolds number is the same for both cases: $R e=1450$. Pictures have been taken at $t^{*}=13.3$ with an exposure time $2 \mathrm{~s}$. We note a strong difference in the dynamics. For $C=0.2$ (deep water), motion is very weak and disorganized in small scale structures. For $C=3.3$ (shallow water), the main flow is a nearly symmetric dipole with two counter-rotative vortices. The vortex cores are not well defined but the global laminar structure is easily identified. One can note that dipoles generated in shallow water are not always symmetric. Their trajectories are variable: symmetric dipoles move straight forward while asymmetric dipoles move along circles. A detailed presentation of a quasi-symmetric dipole is exposed in section.

All these qualitative observations have shown that the shallow water dynamics is very different from the deep water dynamics. In shallow water the vortex dynamics has a Q2D behavior, with mainly horizontal motion and formation of large coherent vortex structures.

\subsection{Reynolds number influence}

We have shown a strong qualitative influence of the confinement number $C$. In order to complete the parametrical study, we have varied the second independent dimensionless number: the Reynolds number $R e$ based on the injected momentum flux.

We have described a range from $R e=1000$ to $R e=1800$. In this range, we have not identified any obvious qualitative influence of $R e$ : large structures appear when $C$ is large, regardless of the Reynolds number.

\section{Quantitative results}

Quantitative measurements have been performed to confirm the influence of $C$ on the vortex dynamics.

We define Cartesian coordinates $x, y$ (horizontal directions) and $z$ vertical direction. Associated velocity components are respectively $u, v$ and $w$, and $E_{x}, E_{y}$ and $E_{z}$ are respectively the kinetic energies associated to $u, v$, and $w$. Every measurement 


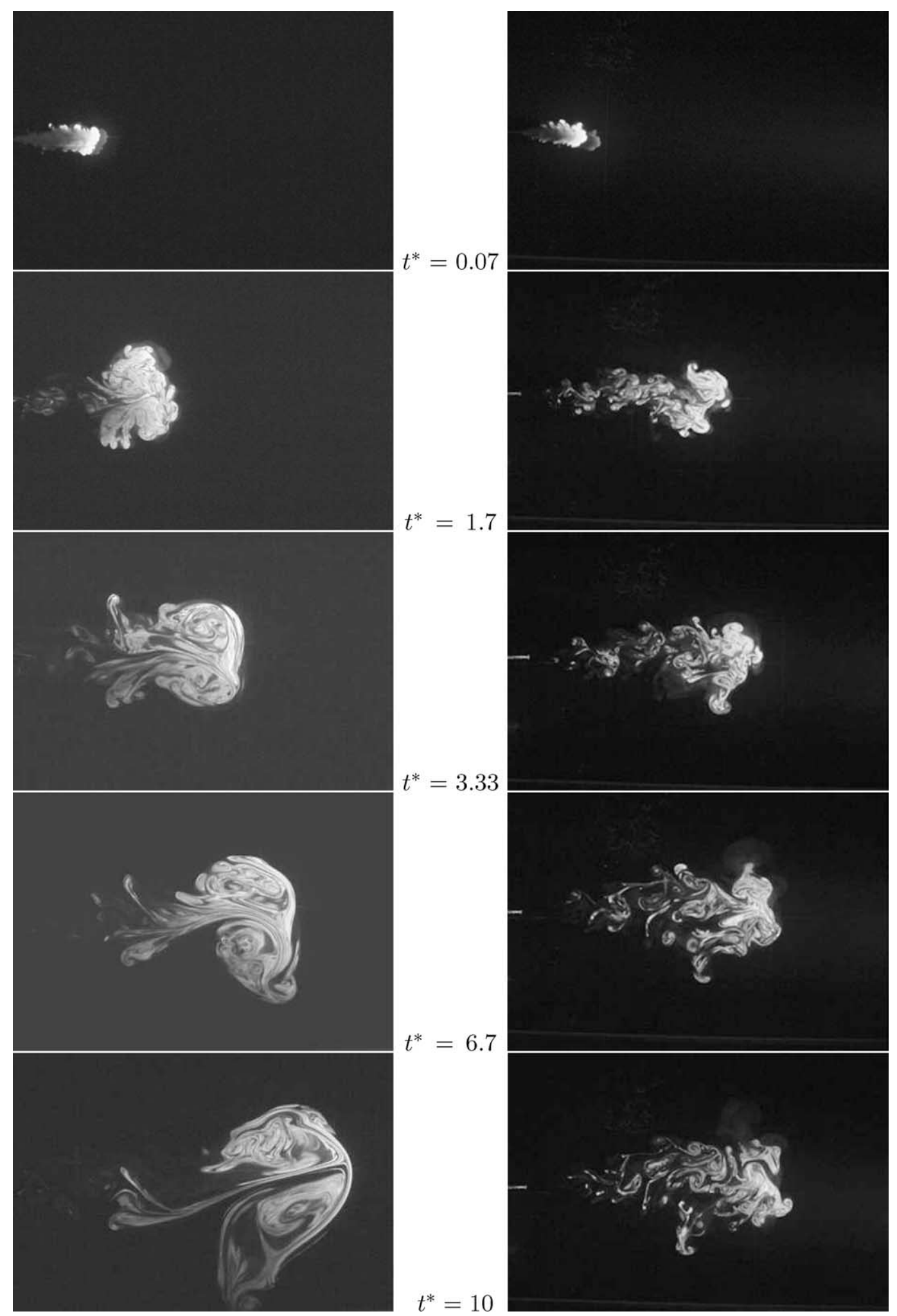

Fig. 2. Compared evolution of a turbulent pulsed jet $(R e=1300)$ in horizontal section, left column corresponds to $C=3$ (shallow water) and right column to $C=0.18$ (deep water). 

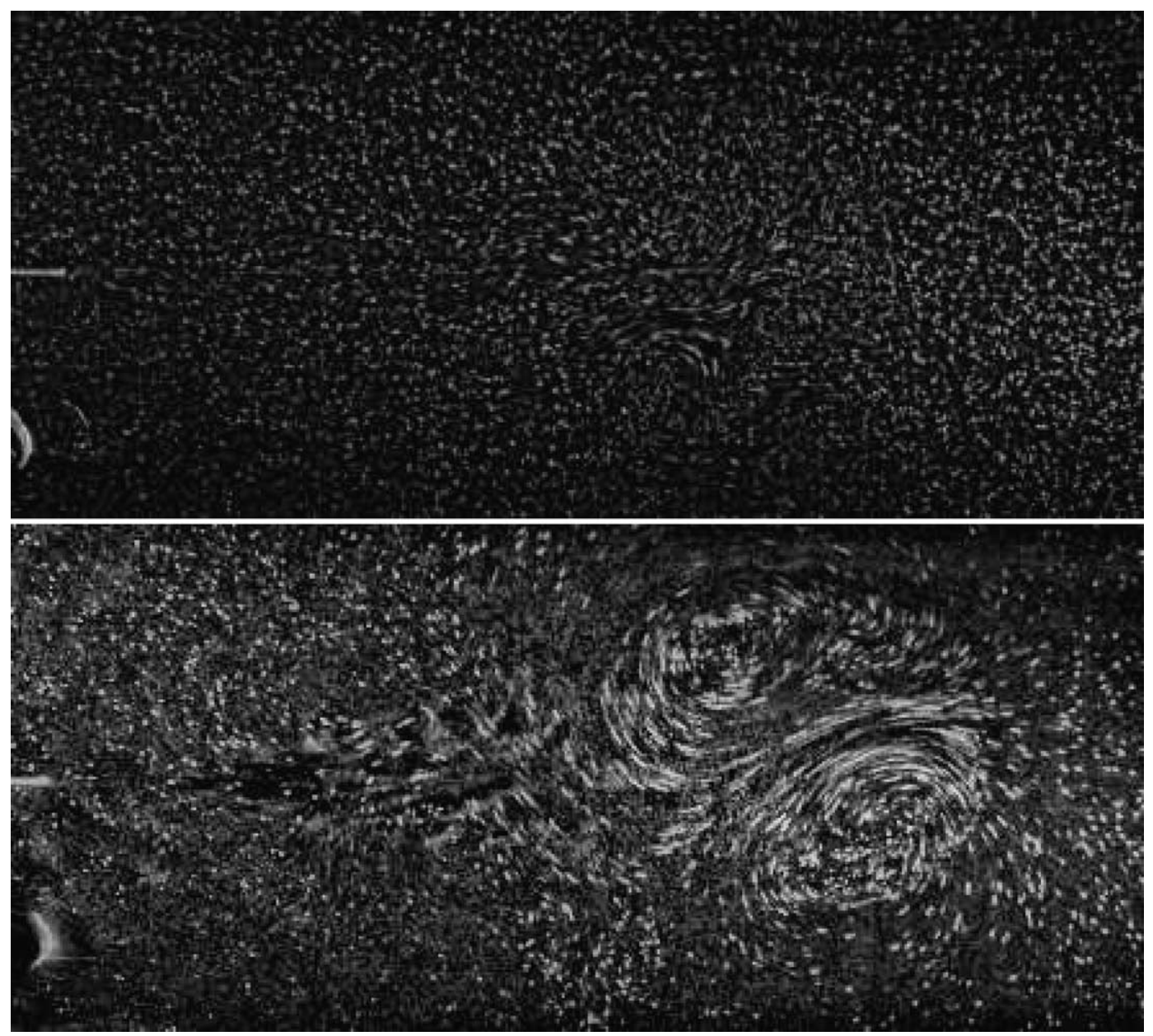

Fig. 3. Particles streaks for $C=0.2$ (upper picture) and $C=3.3$ (lower picture), $R e=1450$, (horizontal section, exposure time $2 \mathrm{~s}$ ).

has been done at $t^{*}=t / t_{\mathrm{inj}}=6$ after the injection. On one hand at this time, the difference between the dynamics in shallow and deep water is well developed and can be easily quantified. On the other hand, whatever the experimental conditions are, the flow remains far from the tank boundaries and can be considered in an infinite domain in the horizontal directions. For $t^{*}>6$, the dynamics keep the same behavior. As shown in Fig. 2, in deep water, the flow is progressively damped until the total motion dissipation. In shallow water, the pulsed jet develop in a dipolar structure which size increases during time. These Q2D dipoles have a travelling motion until they reach the tank boundaries.

In the quantitative results presented in Figs. 4 and 5, each point is an average between two measurements. The vertical bars correspond to the global measurement uncertainties (deviations to the average value and PIV technique uncertainties). Every measurement has been carried out at the following $C$ values: $0.5,1,1.5,2,2.5,3,3.5,4,4.5,5$ and 7 . However, for visual commodity, a slight shift has been added to the $C$ values for the cases $R e=1500$ and $R e=1800$, respectively 0.05 and 0.1 .

\subsection{Comparison of kinetic energies}

Vertical velocity fields are given by PIV measurement on a vertical $(x, z)$ section of the flow along the jet axis $\left(y=l_{t} / 2\right)$. From these velocity fields, we compute two kinetic energies $E_{x}$ and $E_{z}$, respectively defined as the integrals of $u^{2}$ and $w^{2}$ over the plane $(x, z)$ and multiplied by the width of the tank in the $y$ direction $l_{t}$.

$$
E_{x}=\frac{1}{2} l_{t} \int_{S} \rho u^{2} \mathrm{~d} S, \quad E_{z}=\frac{1}{2} l_{t} \int_{S} \rho w^{2} \mathrm{~d} S,
$$

where $S$ is the studied area in the plane $(x, z)$ and $\mathrm{d} S$ the surface element of the PIV meshgrid. 


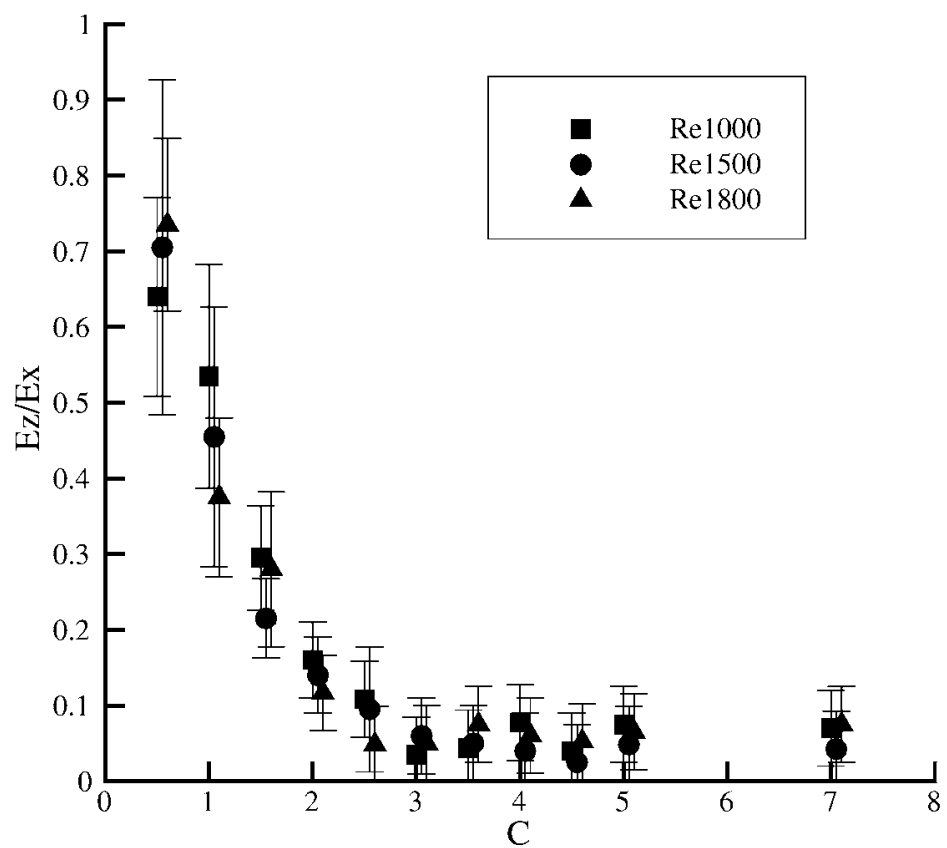

Fig. 4. Evolution of $E_{z} / E_{x}$ vs $C$ for $R e=1000,1500$ and 1800 (at $t^{*}=t / t_{\text {inj }}=6$ ).

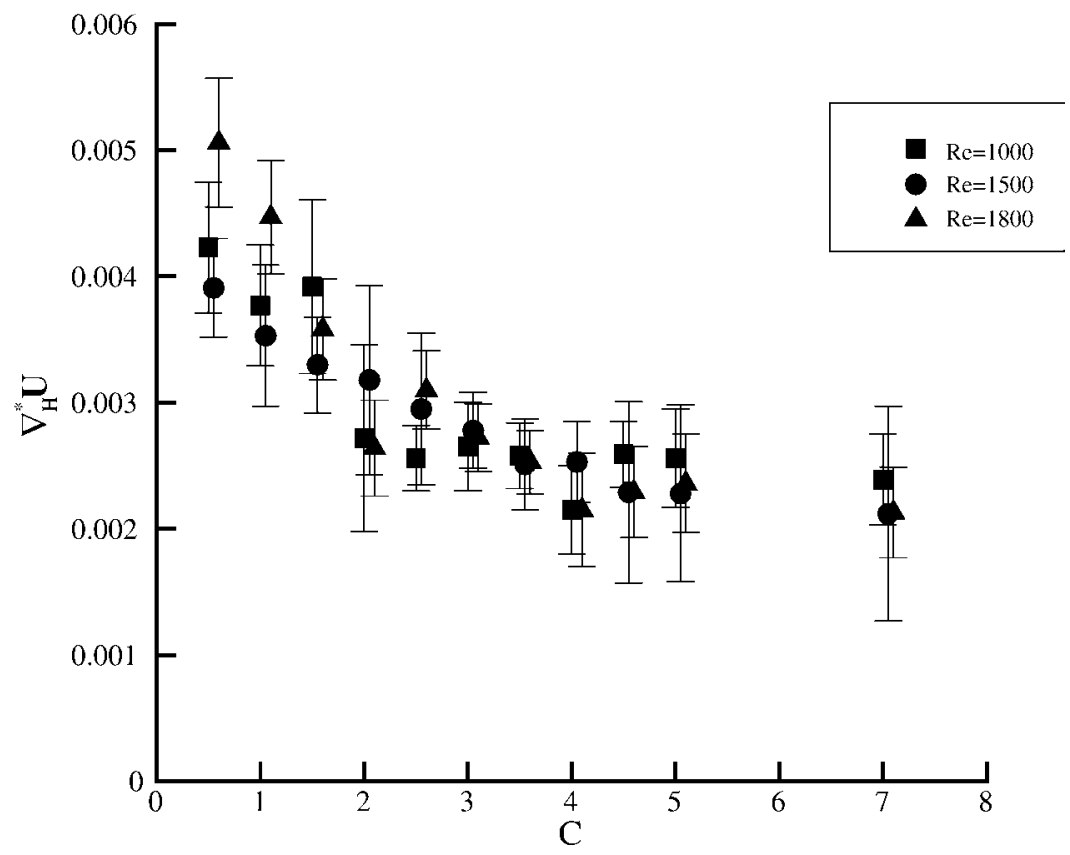

Fig. 5. Evolution of the dimensionless horizontal divergence $\nabla_{H}^{*} U$ vs $C$ for $R e=1000,1500$ and 1800 (at $t^{*}=6$ ).

The ratio $E_{z} / E_{x}$ is then plotted as a function of $C$ in Fig. 4 for three different Reynolds numbers. We first observe that, due to the initial jet orientation, $E_{z} / E_{x}$ is less than 1 for every $C$. We note a global decrease of the ratio $E_{z} / E_{x}$ when $C$ increases. When $C$ is close to $0, E_{z} / E_{x}$ is greater than 0.5 , vertical motion remains intense. For $0<C<2, E_{z} / E_{x}$ decreases rapidly to less than 0.2 . From $C=2$, the decrease stops progressively, and beyond $C=3, E_{z} / E_{x}$ remains inferior to 0.1 . 


\subsection{Horizontal divergence}

Since the flow is incompressible, the divergence of the velocity field is nil. The vertical gradient of velocity vertical component $\partial w / \partial z$ can then be computed from the "horizontal" divergence $\nabla_{H} U$ :

$$
\frac{\partial w}{\partial z}=-\nabla_{H} U=-\frac{\partial u}{\partial x}-\frac{\partial v}{\partial y} .
$$

The horizontal components of the velocity $u$ and $v$ are extracted from PIV measurements in the horizontal $(x, y)$ plane, at half depth $z=H / 2$. The intensity of $\partial w / \partial z$ is representative of the perturbation of the flow quasi-two-dimensionality. In order to quantify this perturbation for different $C$ and $R e$, the average of $|\partial w / \partial z|$ is computed on the whole velocity field and multiplied by $H / U_{\text {inj }}\left(U_{\text {inj }}\right.$ is the injection speed) to present a dimensionless result, called $V_{H}^{*} U$ :

$$
\nabla_{H}^{*} U=\frac{\sum|\partial w / \partial z|}{n_{\mathrm{pts}}} \frac{H}{U_{\mathrm{inj}}},
$$

where $n_{\mathrm{pts}}$ is the number of points of the PIV meshgrid. The evolution of $\nabla_{H}^{*} U$ versus $C$ for three different Reynolds numbers is presented in Fig. 5.

As shown in the previous measurements, the results are strongly dependent of $C$ whereas $R e$ has no influence: $V_{H}^{*} U$ globally decreases when $C$ increases. Until $C=2, \nabla_{H}^{*} U$ decreases rapidly during the transition from a deep to a shallow water layer. Beyond $C=2$, the decrease rate is lower and $\nabla_{H}^{*} U$ reaches progressively a nearly constant value corresponding to the shallow water behavior.

\subsection{Conclusion}

An important influence of the $C$ parameter on the dynamics is highlighted from Figs. 4 and 5: a strong damping of vertical motions appears when $C$ increases. The Reynolds number has no influence in the studied range $(1000<R e<1800)$. Three regimes of the flow can be distinguished. When $C<1$, the water layer is deep, motion is isotropic. Between $C=1$ and $C=2$, a transition zone occurs where the confinement progressively constrains the flow, but the vertical motion remains significant. For $C>2$ a particular behavior appears where the flow is mainly Q2D. Coherent horizontal vortex dipoles, which sizes are large compared to depth, are observed. The fluid layer can then be considered shallow. However, vertical perturbations remains in the flow $\left(E_{z}, \nabla_{H} U \neq 0\right)$ in the range we have studied $(2<C<8)$. The structure of these vertical motions is detailed in the following section.

\section{Dipole characteristics}

As we have shown in previous sections, dipoles can be generated from horizontal pulsed jets in a shallow water layer. We present here the main features of a dipole generated with $C=3$ and $R e=1300$, which is representative of all other dipoles observed during our experiments.

Velocity field is presented in Fig. 6 . The longest vector represents a $1.9 \mathrm{~cm} / \mathrm{s}$ velocity. The observed area is $0.46 \times 0.35 \mathrm{~m}^{2}$. The measurements are done at $t^{*}=t / t_{\mathrm{inj}}=7.5$. We can note the weak asymmetry of the structure. The front of the dipole is characterized by a sharp decay of the horizontal velocities, as seen previously in Fig. 3. It is confirmed by the vertical section of the flow shown in Fig. 7. In the main part of the dipole, particles streaks are long and quasi-horizontal. In this zone, the flow is laminar and quasi-two-dimensional, with very weak vertical motions. Observations show the stability of the boundary layer on the bottom, in the main part of the dipole. This is confirmed by the boundary layer Reynolds number calculation. The balance between the diffusion and advection time of the boundary layer (thickness $\delta$ ) leads to:

$$
\frac{\delta^{2}}{v} \approx \frac{a}{U} .
$$

Here $a$ is the dipole horizontal length scale. The boundary layer thickness can be written:

$$
\delta \approx \sqrt{\frac{\nu a}{U}} .
$$

With the typical values $U=0.01 \mathrm{~m} / \mathrm{s}$ and $a=0.2 \mathrm{~m}$, the boundary layer Reynolds number is then:

$$
\operatorname{Re}_{\delta}=\frac{U \delta}{v}=\sqrt{\frac{U a}{v}} \approx 45
$$




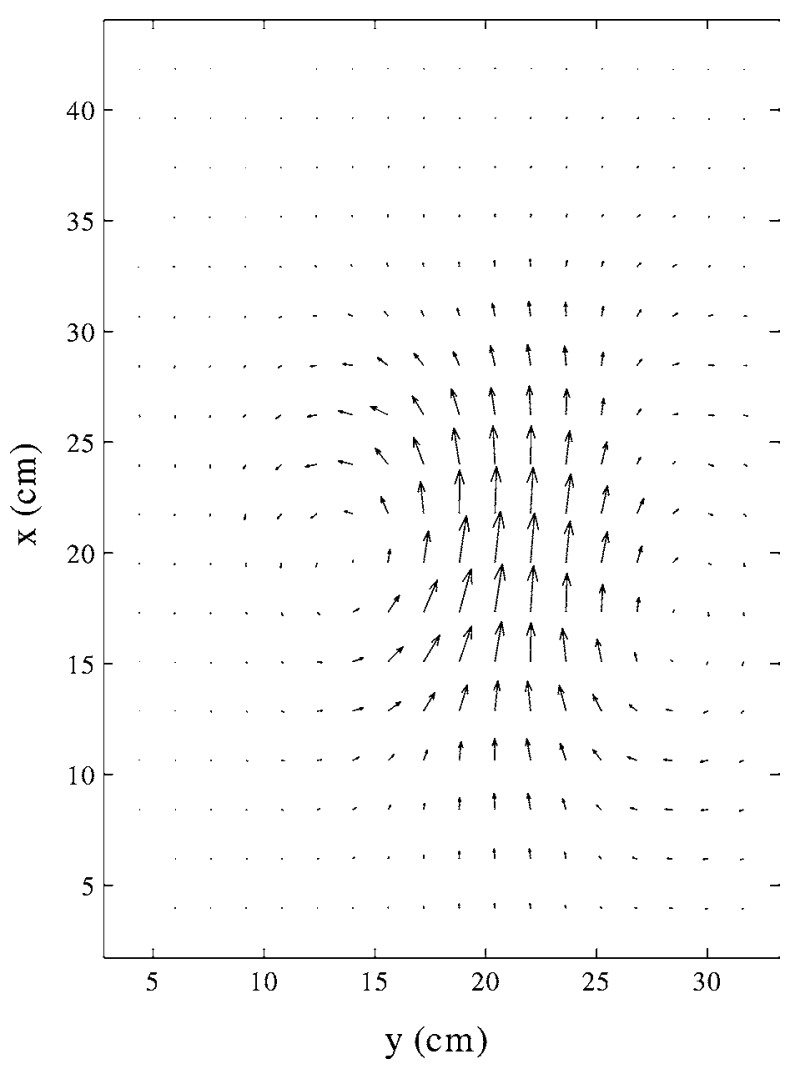

Fig. 6. Horizontal velocity field for $C=3$ and $R e=1300$ at $t^{*}=7.5$, the longest vector intensity is $1.9 \mathrm{~cm} / \mathrm{s}$.

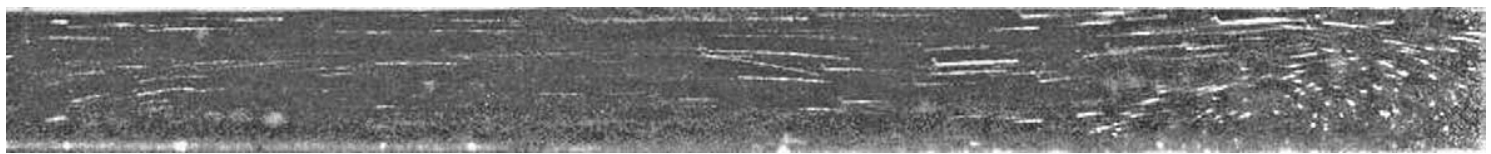

Fig. 7. Dipole in axial vertical section $\left((x, z)\right.$ plane), for $C=3$ (shallow water) and $R e=1300$ at $t^{*}=7.5$. Observation area is $25 \mathrm{~cm}$ long and $2 \mathrm{~cm}$ high.

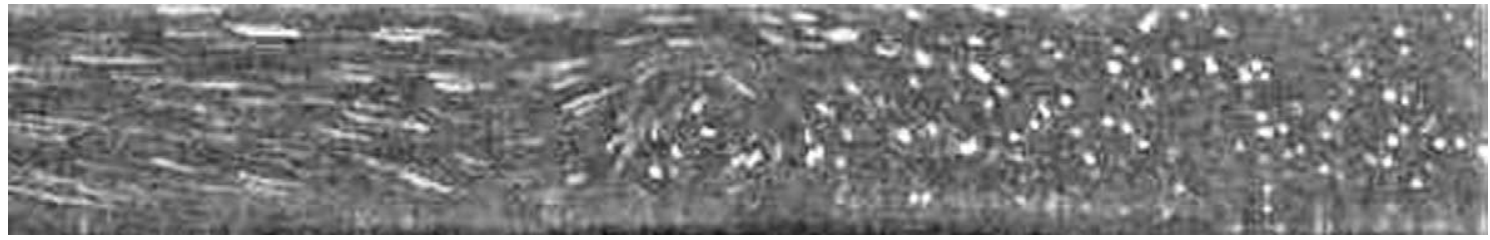

Fig. 8. Frontal circulation streaks in axial vertical section $((x, z)$ plane), for $C=3$ (shallow water), $R e=1300$ at $t *=7.5$. Observation area is $12.2 \mathrm{~cm}$ long and $2 \mathrm{~cm}$ high.

The value is far from the critical value $R e_{\delta}=350$ from which the boundary layer becomes unstable [22]. At the dipole front, horizontal velocity components decrease in the streamwise direction while vertical motions increase. Particles streaks reveal a vertical circulation in the dipole front, probably caused by the shear induced by the no-slip condition on the glass bottom. A more detailed picture is presented in Fig. 8. This vertical circulation takes place all over the depth. It is the main responsible of the presence of the vertical motion measured in the dipole (Figs. 4, 5).

Theoretical model of purely 2D steady dipole assumes a linear relationship between the vorticity and the stream function inside the dipole [17,18]. Outside the dipole, which has a circular shape of radius $a$, the flow is irrotational and similar to a potential flow around a circular cylinder. Experiments on dipoles in stratified [15] or electromagnetically driven flows [19] have 
shown in most cases a nonlinear relationship $\omega=f(\psi)$ (where $\omega$ is the vertical vorticity and $\psi$ is the stream function). In every cases, the relationship derivative $f^{\prime}$ is constant or has a singular maximum at the vortex core. In our experiments in shallow water, the dipoles are characterized by a specific feature: the presence of a vertical circulation in the dipole front. This circulation disturbs the flow two-dimensionality and it is of great interest to known if the $\omega / \psi$ relationship of dipole in shallow water is linear. For latter comparison, it is useful to mention general characteristics of the 2D dipole model. First the maximum velocity (in the dipole framework) is given by $U_{m}=2.49 U_{0}$ where $U_{0}$ is the translation speed. In the laboratory frame, this speed is then $U_{m}^{*}=U_{m}+U_{0}=3.49 U_{0}$. Furthermore, the extremal vorticity in the dipole is equal to $\omega_{\max }=\left|\omega_{\min }\right|=2.9 k U_{0}\left(k^{2}\right.$ is the proportionality coefficient between $\omega$ and $\left.\psi: \omega=k^{2} \psi\right)$ and the distance between the points of minimum and maximum vorticity is $d_{v}=0.96 a$.

By numerical differentiation of the velocity field according to

$$
\omega=\frac{\partial v}{\partial x}-\frac{\partial u}{\partial y}
$$

one obtains the value of the vorticity $\omega$ in each grid point. Next, the stream function $\psi$ is calculated by numerically solving the following Poisson-type equation:

$$
\omega=-\nabla^{2} \psi
$$

The stream-function is only defined for purely 2D flows. The dipoles observed in the present experiments are characterized by significant vertical motion. However, we can assume a scale-separation: a main horizontal flow at large length scale which greatly exceeds the flow depth and a secondary vertical motion in the frontal circulation, with a length scale close to the depth. The main flow is a 2D dipole, which omega-psi relationship can be computed. In order to compare with the steady dipole model, vorticity and stream function are computed from the horizontal velocity field in a frame of reference moving with the dipole. For a dipole translating with velocity $\left(U_{x}, U_{y}\right)$ this requires the following transformation: $\psi^{\prime}=\psi-U_{x} y+U_{y} x$, while the vorticity remains unchanged. In the following, $\psi^{\prime}$ will be simply called $\psi$. The translation speed is determined by comparing the positions of the vortex centers on two frames, separated with the delay time $\delta t=3.33 \mathrm{~s}$. Uncertainties obtained with this method are inferior to $8 \%$. The translation velocity is equal to $U_{0}=\sqrt{U_{x}^{2}+U_{y}^{2}}=0.57 \pm 0.045 \mathrm{~cm} \mathrm{~s}^{-1}$ and the maximum velocity reached between the vortex centers is $U_{m}^{*}=1.9 \pm 0.152 \mathrm{~cm} \mathrm{~s}^{-1}$. Thus $U_{m}^{*}=3.33 U_{0}$, which is slightly inferior to the theoretical model prediction. Typical results of vorticity calculation and stream function with translation correction are shown in Figs. 9 and 10. These figures clearly reveal the asymmetric structure of the dipole in shallow water. The shape is not circular, the dipole is elongated in the translation direction. Due to this shape and the asymmetry of the dipole, it is difficult to determine the dipole radius. However, one can estimate from the streamlines a "transverse" radius $a_{\mathrm{tv}}=9.2 \mathrm{~cm} \pm 0.46$. From the vorticity contour plots, the distance between the extremal vorticity is measured at $d_{\mathrm{v}}=12.2 \mathrm{~cm} \pm 0.61$. One obtain $d_{\mathrm{v}} / a_{\mathrm{tv}}=1.32 \pm 0.132$, which is greater than the model value $d_{\mathrm{v}} / a=0.96$. This means that the vortices are more separated than in the theoretical model or in the stratified fluid experiments. This difference in linking indicates apparently a more pronounced entrainment of ambient fluid. The frontal circulation has probably a preponderant role in this greater entrainment.

The $\omega / \psi$ relationship is presented in Fig. 11. The points clustered in the horizontal band with $\omega=0$ all lie outside the dipolar vortex, and represent the exterior potential flow. The two branches with $\omega>0$ and $\omega<0$ each represent one half of the dipole. Although some scatter is observed, the points collapse quite well into a single curve. Experimental results are compared with a linear relationship $\omega=-k^{2} \psi\left(k^{2}=0.41\right)$ and a sinh-relationship $(\omega=-0.26 \sinh 1.41 \psi)$. Better agreement is found with the sinh-relationship, approached in the present experiments by the cubic polynomial $\omega=a \psi-b \psi^{3}(a=-0.31$ and $b=-0.11)$. In their statistical-mechanics approach of characterizing steady-state structures in 2D flows, based on searching for the most probable state, Joyce et Montgomery [23] found evidence for the validity of this sinh-relationship for such 2D flow structures. Experiments on dipoles in stratified fluids [15], MHD [19] or in soap films [20] have revealed the same relationship. Theoretical study carried out by Pasmanter [24] has proposed a relationship of the form:

$$
\omega=-c \frac{\psi}{d^{2}-\psi^{2}}
$$

With $c=-1.4$ and $d=-1.95$ this relationship is not distinguishable from the sinh relationship in the studied interval. Thus, despite of the presence of a vertical circulation in the front of the dipoles observed in shallow water layer and a noncircular asymmetric shape, the $\omega / \psi$ relationship is close to those observed in their counterparts. In the linear model, the intensities of vorticity extrema are given by: $\omega_{\max }=\left|\omega_{\min }\right|=2.9 \mathrm{k} U_{0}$. In our experiments, the vorticity extrema are not symmetric. The positive extremum is $\omega_{\max }=0.45 \mathrm{~s}^{-1}$ and the negative one is $\omega_{\min }=-0.3 \mathrm{~s}^{-1}$. If we use the coefficient $k^{2}=0.41$ found to be the best one for a linear approximation of the $\omega / \psi$ relationship, the theoretical vorticity should be $\omega_{\max }=0.67 \mathrm{~s}^{-1}$. The "smoother" vorticity observed in the experiments confirms the greater entrainment of ambient fluid which occurs the dipoles in shallow water. The frontal circulation has probably an important role in this entrainment increase. 


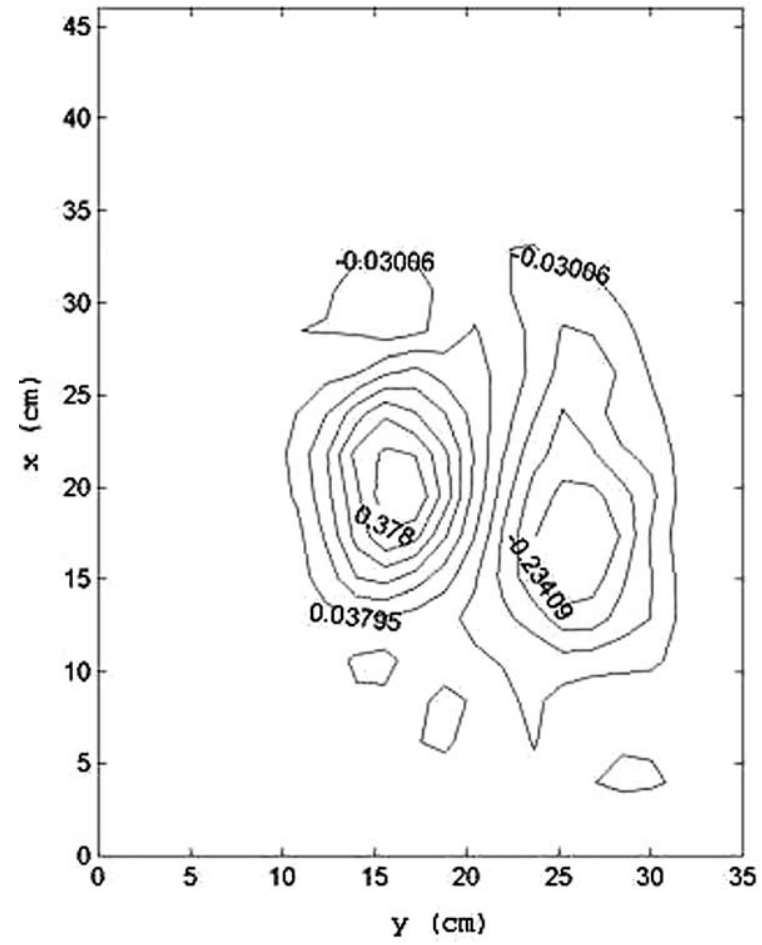

Fig. 9. Vorticity distribution $\left(\mathrm{s}^{-1}\right)$ in the horizontal $(x, y)$ plane at $z=H / 2$ for $C=3$ and $R e=1300$ at $t^{*}=7.5$.

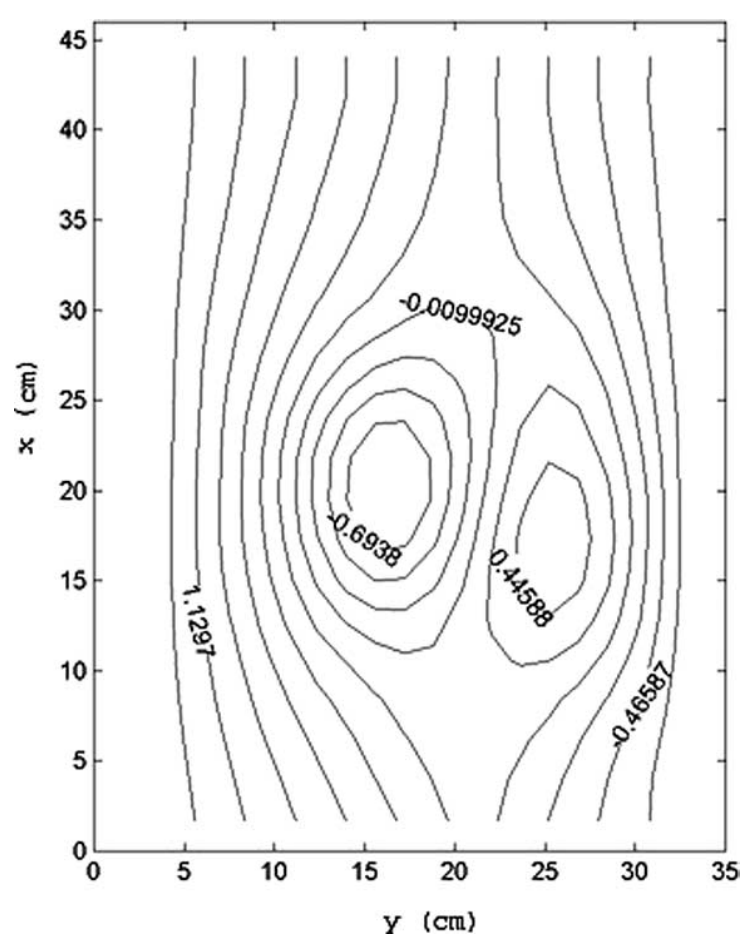

Fig. 10. Stream function distribution $\left(\mathrm{cm}^{2} \mathrm{~s}^{-1}\right)$ in the horizontal $(x, y)$ plane at $z=H / 2$ for $C=3$ and $R e=1300$ at $t^{*}=7.5$.

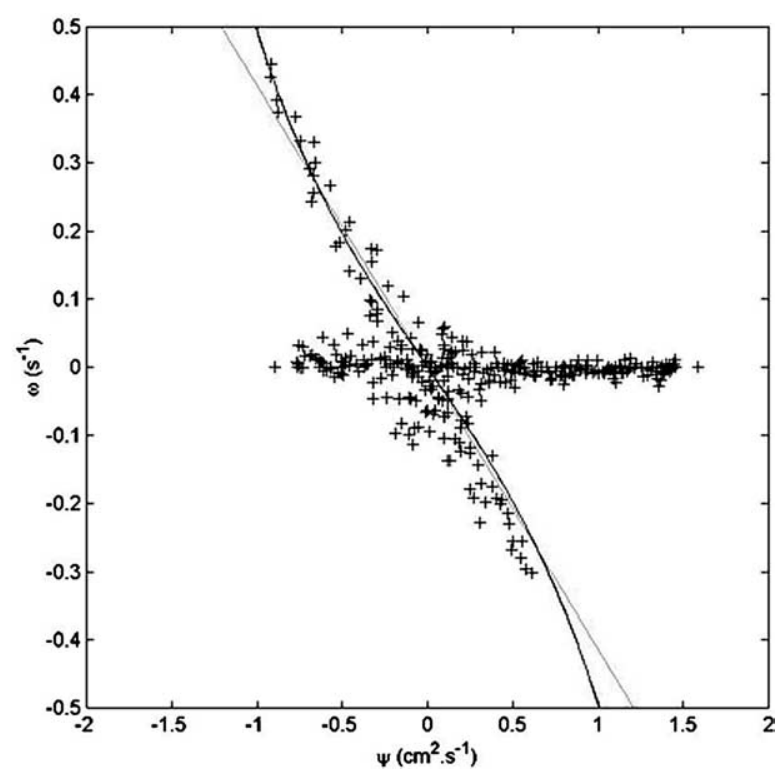

Fig. 11. Vorticity-stream function relationship for $C=3$ and $R e=1300$ at $t^{*}=7.5$, compared to $\omega=-0.41 \psi$ for the linear case (gray) and to $\omega=-0.26 \sinh (1.41 \psi)$ for the nonlinear case (black).

\subsection{Conditions of quasi-two-dimensionality}

As exposed in the introduction, the quasi-two-dimensionality condition for shallow fluid layers $\left(R e_{\lambda} \ll R e_{\nu}\right)$ proposed by Dolzhanskii for shear flows [10] is equivalent to $H^{2} / 2 L^{2} \ll 1$ (where $H$ is the depth and $L$ the horizontal length scale). If 
we consider the typical values $H=2 \mathrm{~cm}$ and $L=20 \mathrm{~cm}$ for a dipole observed during our experiments, this ratio $H^{2} / 2 L^{2}$ is $5 \mathrm{e}-3$. The quasi-two-dimensionality condition in a shallow fluid layer is fulfilled by the dipoles generated with $C>2$. During the dipoles evolution, the horizontal length scale $L$ increases by viscous diffusion and fluid entrainment while the depth $H$ remains constant, so that the theoretical two-dimensionality should increase over time. However, our observations have shown the persistence of a frontal vertical circulation.

In their numerical simulations on monopolar vortices in a shallow water carried out by Satijn et al. [11], the quasi-twodimensionality of the flow is studied depending on two dimensionless parameters: $R e$ and $R e_{\lambda}$. They are based on the typical vorticity $\omega: \operatorname{Re} \lambda=\omega / \lambda$ (where $\lambda=\pi^{2} v / 4 H^{2}$ is the external friction parameter with $H$ the height of the layer and $v$ the kinematical viscosity) and the structure Reynolds number $R e=L^{2} \omega / v$ ( $L$ represents the typical horizontal length scale in the flow). If we consider the typical vorticity of the dipole in a shallow water layer as $\omega=U / 2 L$, where $U$ is the characteristic velocity (typical value $0.01 \mathrm{~m} / \mathrm{s}$ ), the parameters proposed by Satijn take the following values: $R e \approx 1000$ and $R e_{\lambda} \approx 4$. In the regime diagram presented by Satijn et al., these values correspond to a Q2D flow. However, due to the different vortex structures (monopole and dipole), the direct comparison between our experiments and the numerical simulation should be made with caution.

These quasi-two-dimensionality conditions for a thin fluid layer are theoretically fulfilled by shallow water dipoles. However, observations and measurements have shown significant 3D motions in a vertical circulation which takes place all along the dipole front. The shear generated upon the bottom is responsible for such motions, but is not strong enough to destabilize the boundary layer, in the main part of the flow.

\section{Conclusions}

This paper outlines an experimental study of vortex dynamics in a shallow water layer. Our aim was to identify a transition from fully 3D behavior (deep fluid layer) to Q2D behavior (shallow water layer). This transition has been studied by imposing a progressive vertical confinement on an axisymmetric horizontal turbulent impulsive jet. The jet evolution has been studied depending on two dimensionless parameters: the Reynolds number $R e$ and the confinement number $C$. They are defined by:

$$
R e=\frac{\sqrt{Q}}{v}, \quad C=\frac{\sqrt{Q}}{H^{2}} t_{\mathrm{inj}},
$$

where $v$ is the kinematic viscosity, $Q$ the injected momentum flux, $H$ the water depth and $t_{\text {inj }}$ the injection duration.

Every measurement has been performed from dimensionless times between $t^{*}=t / t_{\text {inj }}=6$ and $t^{*}=7.5$, which are small compared to the time necessary to the total viscous dissipation of motions. Qualitative and quantitative experimental investigations have shown the preponderant influence of $C$ on the jet development whereas the Reynolds number has no influence between $R e=1000$ and $R e=1800$. When $C<1$, the jet spreading is free. The generated turbulence remains fully $3 \mathrm{D}$ and is not constrained by the boundaries. When $C$ increases $(1<C<2)$, the geometric and kinematic conditions imposed by the bounding surfaces start to influence the development of the pulsed jet in a distinct way. From $C=2$, a particular behavior systematically appears. It is characterized by a damping of the vertical motion and formation of large horizontal vortex structures, mainly dipoles. A detailed study of the dipoles characteristics has shown the main features: a quasi-two-dimensional laminar main flow, a vertical circulation in the dipole front and the nonlinearity of the $\omega / \psi$ relationship.

However, although the dipole dynamics are close to Q2D flows, vertical motion occurs systematically in our experiments. It consists in a vertical circulation in the front of the dipole induced by the no-slip condition on the bottom. In order to avoid this slip condition, we have performed experiments with a heavier fluid layer on the tank bottom. Our qualitative observations have revealed the quasi-two-dimensional structure of the flow, without any vertical circulation at the dipole front [25]. In geophysical dipole vortices, observed for instance in the head of rip currents [4], the presence of a vertical circulation which interacts strongly with the bottom and leads to an increase of fluid entrainment will have a preponderant role in sediment transport and mixing processes.

Further experimental investigations at larger scale have been carried out to generate turbulent vortex dipoles in a shallow water layer. They will be the subject of a future paper.

This work was supported by the Délégation Generale pour l'Armement.

\section{References}

[1] W.S.J. Uijttewaal, R. Booij, Effects of shallowness on the development of free-surface mixing layers, Phys. Fluids 12 (2) (2000) $394-402$.

[2] J.C. Lin, M. Orzogen, D. Rockwell, Space-time development of the onset of a shallow-water vortex, J. Fluid Mech. 485 (2003) $33-66$.

[3] G.H. Jirka, Large scale flow structures and mixing processes in shallow flows, J. Hydraulic Res. 39 (6) (2001) 567-573. 
[4] J.A. Smith, J.L. Largier, Observations of nearshore circulations: rip currents, J. Geophys. Res. 100 (1995) 10967-10975.

[5] N. Drønen, H. Karunarathna, J. Fredsøe, B. Mutlu Sumer, R. Deigaard, An experimental study of rip channel flow, Coast. Eng. 45 (2002) $223-238$.

[6] T. Dracos, M. Giger, G.H. Jirka, Plane turbulent jets in a bounded fluid layer, J. Fluid Mech. 241 (1992) 587-614.

[7] D. Chen, G.H. Jirka, Experimental study of plane turbulent wakes in a shallow water layer, Fluid Dynamics Res. 41 (1995) 11-41.

[8] R. Booij, J. Tukker, Integral model of shallow mixing layers, J. Hydraulic Res. 39 (2) (2001) 169-179.

[9] W.S.J. Uijttewaal, V. Weitbrecht, C.F. Graf von Carmer, G.H. Jirka, Experiments on Shallow Flows Turbulence, in: Proc. 2001 International Symposium on Environmental Hydraulics, 2001.

[10] F.V. Dolzhanskii, V.A. Krymov, D.Yu. Manin, An advanced experimental investigation of quasi-two-dimensionnal shear flows, J. Fluid Mech. 241 (1992) 705-722.

[11] M.P. Satijn, A.W. Cense, R. Verzicco, H.J.H. Clercx, G.J.F. van Heijst, Three-dimensional structure and decay properties of vortices in shallow fluid layers, Phys. Fluids 13 (2001) 1932-1945.

[12] E.J. Hopfinger, G.J.F. van Heijst, Vortices in rotating fluids, Ann. Rev. Fluid Mech. 25 (1993) 241-289.

[13] O.U. Velasco Fuentes, G.J.F. van Heijst, Experimental study of dipolar vortices on a topographic $\beta$-plane, J. Fluid Mech. 259 (1994) 79-106.

[14] L. Zavala Sansòn, G.J.F. van Heijst, N.A. Backx, Ekman decay of a dipolar vortex in a rotating fluid, Phys. Fluids 13 (2001) $440-451$.

[15] J.B. Flor, G.J.F. van Heijst, An experimental study of dipolar vortex structures in a stratified fluid, J. Fluid Mech. 279 (1994) 101-133.

[16] S.I. Voropayev, Ya.D. Afanasyev, I.A. Filippov, Horizontal jets and vortex dipoles in a stratified fluid, J. Fluid Mech. 227 (1991) $543-566$.

[17] H. Lamb, Hydrodynamics, 6th edition, Dover, 1932.

[18] V.V. Meleshko, G.J.F. van Heijst, On Chaplygin's investigations of two-dimensional vortex structures in an inviscid fluid, J. Fluid Mech. 272 (1994) 157-182.

[19] J.M. Nguyen Duc, J. Sommeria, Experimental characterization of steady two-dimensional vortex couples, J. Fluid Mech. 192 (1988) 175-192.

[20] Y. Couder, C. Basdevant, Experimental and numerical study of vortex couples in two-dimensional flows, J. Fluid Mech. 173 (1986) 225-251.

[21] H. Schlichting, Boundary Layer Theory, Mc Graw-Hill, New York, 1979.

[22] G.K. Batchelor, An Introduction to Fluid Dynamics, First Cambridge Mathematical Library edition, 2000.

[23] G. Joyce, D.C. Montgomery, Statistical mechanics of negative temperature states, Phys. Fluids 13 (1973) 1139-1145.

[24] R.A. Pasmanter, On long lived vortices in 2-D viscous flows, most probables states of inviscid 2-D flows and a soliton equation, Phys. Fluids 6 (1994) 1236-1241.

[25] D. Sous, Dynamique tourbillonnaire en milieu peu profond, $\mathrm{PhD}$ thesis, Université Bordeaux 1, 2003. 